\title{
Problem-solving interventions and depression among adolescents and young adults: A systematic review of the effectiveness of problem-solving interventions in preventing or treating depression among 13-25 year olds
}

Jane Lewis ${ }^{1}$, Jade Mitchell ${ }^{1}$, Sangita Chakraborty ${ }^{1}$, Bryce D. McLeod ${ }^{2}$, Kristina L. Metz ${ }^{3}$, Ludvig Bjørndal ${ }^{1}$, Robyn Mildon ${ }^{1}$ and Aron Shlonsky ${ }^{4}$

${ }^{1}$ Centre for Evidence and Implementation, UK and Australia

${ }^{2}$ Department of Psychology, Virginia Commonwealth University, USA

${ }^{3}$ Bloomberg School of Public Health, Johns Hopkins University, USA

${ }^{4}$ Department of Social Work, Monash University, Australia

This review was produced with the support of a grant from Wellcome. Correspondence should be addressed to Jane Lewis, Centre for Evidence and Implementation, email jane.lewis@ceiglobal.org

This work is licensed under a Creative Commons Attribution 4.0 International License which permits unrestricted use, distribution and reproduction provided the original work is properly cited.

\section{ABSTRACT}

Background: Problem-solving (PS) has been identified as a common element in multiple evidence-based treatments for the prevention and treatment of depression. We undertook a systematic review of evidence for the effectiveness and implementation of PS for the prevention and treatment of depression among 13-25 year olds.

Methods: We searched electronic databases (Psyclnfo, Medline, and Cochrane Library) for studies published between 2000 and 2020 described by authors as a PS intervention or including PS approaches to treat or prevent depression in 13-25 year olds. Eighteen out of 902 effectiveness studies and 1 out of 480 implementation studies met inclusion criteria.

Results: The interventions studied were heterogeneous in population, intervention, modality, comparison condition, study design, and outcome. Eight1 focused purely on PS; eight used PS as part of a wider intervention. Six studies found positive effects in reducing depression, and two in reducing suicidality. Seven measured effects on PS skills. There was little evidence of

\footnotetext{
${ }^{1}$ Addressed in ten studies
} 
change in PS skills, about PS skills as mediator or moderator of effects on depression, or about the fit, feasibility, or acceptability of PS interventions.

Discussion: There is mixed evidence about the effectiveness of PS as treatment and prevention of depression among AYAs although our findings suggest it can work in a wide range of contexts and forms. Possible explanations for limited effectiveness are: not all studies included populations where depression was known to be present; variability in quality, dosage and fidelity monitoring; small samples size and short follow-up periods.

\section{INTRODUCTION}

Depression among adolescents and young adults is a serious, prevalent problem. A striking increase is seen in early adolescence (Dietz, Silk, \& Amole, 2019): rates of depression almost double between the age of 13 (8.4\%) and 18 (15.4\%) (Merikangas et al., 2010), and are highest for adolescents and young adults (AYA; 20-30\%) in low- and middle-income countries (LMIC). A lasting gender gap starts at age 13, with females twice as likely to be diagnosed with a depressive disorder (Merikangas, Nakamura, \& Kessler, 2009). Research also suggests that the mean age of onset for depressive disorders is decreasing and prevalence increasing for AYA.

Depressive episodes for AYA tend to recur and carry into adulthood. Twenty-six percent of adolescents relapse within a year after a depressive episode (Kovacs, Feinberg, Crouse-Novak, Paulauskas, \& Finkelstein, 1984; Lewinsohn et al., 1993) and adolescents experiencing depression are six times more likely to experience episode as an adult (Avenevoli, Knight, Kessler, \& Merikangas, 2008; Zisook et al., 2007). Depressive episodes are associated with significant impairment in academic performance, social problems, higher risk of comorbid disorders, and increased rates of suicide (Angold \& Costello, 2001; Bridge et al., 2008; PuigAntich et al., 1993; Rohde, Lewinsohn, \& Seeley, 1991). Overall, the persistent course, significant impairment, and increased mortality make depression the third leading cause of disability worldwide (Smith, 2014). 
Psychosocial interventions, such as cognitive-behavioural therapy (CBT) and interpersonal therapy (IPT), have shown promise in preventing and treating depression (Stice et al., 2009; Weersing et al., 2017). However, meta-analyses indicate that effect sizes for depression treatments are small to moderate for adolescents (Weisz et al., 2019) and adults (Johnsen \& Friborg, 2015). A similar picture is seen for prevention programmes (Stice et al., 2009).

Up to half of adolescents with depression do not receive treatment (Merikangas et al., 2011), and this rate approaches 90\% in LMIC (Chisholm et al., 2016; Demyttenaere et al., 2004; Saxena et al., 2007). The limited number of AYA receiving treatment, questionable quality of community services, and limited impact of evidence-based treatments (EBTs) suggest that the mental health service system may not be adequately serving individuals with depression.

New strategies are needed to improve accessibility and effectiveness. One strategy is to move away from an emphasis on EBTs (e.g. CBT) to a focus on discrete treatment techniques that demonstrate positive effects across multiple studies that meet certain methodological standards (Chorpita \& Daleiden, 2009). This has the potential to reduce implementation costs and improve sustainment (Hogue et al., 2017), is scalable to LMIC (Chibanda et al., 2011; Eskin, Ertikin, \& Demir, 2008; Perera \& Kathriarchchi, 2011), community delivery (Biggam \& Power, 2002; Spence et al., 2003), and is culturally adaptable.

One potential common element for depression is problem solving (PS). PS trains individual to handle daily stressors, and commonly includes problem identification, problem definition, and the development, evaluation, selection and implementation of solutions. The specification of steps and intensity varies: most common manuals have from four (D'Zurilla and Goldfried, 1971) to seven or more steps (D'Zurilla and Nezu, 2007), with international manuals such as CETA (Murray et al., 2014) having seven steps (with several sub-steps) - see Box 1. It is operationalised in manualized treatments (D'Zurilla \& Nezu, 2007; Spence et al., 2003) and is identified as a common element in multiple prevention (Boustani et al., 2015; Spence, Sheffield, \& Donovan, 2003) and treatment (Bell \& D'Zurilla, 2009; Malouf, Thorsteinsson, \& Schutte, 2006) programmes for depression and other mental health conditions (Chorpita \& Daleiden, 2009; Skeen et al., 2019; Weisz et al., 2017), and thus has transdiagnostic value.

The relationship between PS and depression is complex (Speckens \& Hawton, 2005) and PS may play multiple roles in the association between stress and depression (Nezu 1987). PS may 
moderate the effect of stress on depression (Fry \& Goodman, 2000) and the effectiveness of treatment on depression and suicidality (Becker-Weidman et al., 2010). It may be the causal mechanism by which stress leads to depression or suicidality (Chang, 2002). It is also unclear whether it is PS orientation or PS skills and performance that is most relevant (Becker-Weidman et al., 2010).

\section{Box 1: Operationalisation of PST in two example programmes}

\begin{tabular}{|c|c|c|}
\hline Programme & $\begin{array}{l}\text { Problem Solving Therapy } \\
\text { (D'Zurilla and Goldfried, 1971) }\end{array}$ & $\begin{array}{l}\text { CETA (Common Elements Treatment } \\
\text { Approach) (Murray et al., 2014) }\end{array}$ \\
\hline $\begin{array}{l}\text { Dosage and } \\
\text { coverage }\end{array}$ & $\begin{array}{l}\text { May involve } 8-16 \text { sessions, } \\
\text { focusing on problem solving }\end{array}$ & $\begin{array}{l}\text { May involve } 8-12 \text { sessions covering } \\
\text { relaxation, cognitive coping, exposure- } \\
\text { trauma memories, exposure-live, cognitive } \\
\text { restructuring, behavioural activation and } \\
\text { problem solving }\end{array}$ \\
\hline $\begin{array}{l}\text { Key } \\
\text { elements }\end{array}$ & $\begin{array}{l}\text { - Problem orientation: seeing } \\
\text { problems as a challenge and } \\
\text { solvable } \\
\text { - Problem-solving skills } \\
\text { - Problem definition and } \\
\text { formulation } \\
\text { - Generation of alternative } \\
\text { solutions } \\
\text { - Decision making } \\
\text { - Solution verification: }\end{array}$ & $\begin{array}{l}\text { - Identify the problem } \\
\text { - Identify a goal } \\
\text { - Brainstorm solutions } \\
\text { - Evaluate solutions } \\
\text { - Choose a solution } \\
\text { - Develop an action plan (including } \\
\text { identifying challenges) } \\
\text { - Test and review }\end{array}$ \\
\hline
\end{tabular}

This review is, to our knowledge, the first systematic review of PS interventions for AYA.

\section{YOUNG PEOPLE AND PRACTITIONERS CONVERSATIONS}

To contextualise the review, we held conversations with AYA $(n=14)$ and practitioners providing mental health and psychosocial support (MHPSS) to AYA $(n=10)$ in the United States, United Kingdom, Singapore, Mexico, Myanmar, Lebanon, Ukraine, and Zambia. Conversations were voluntary and facilitated by local staff following guidelines.

Conversations indicated that depression in AYA looks similar across cultures. Onset was typically within early to mid-adolescence (age 10-15), but external factors (e.g., family, peer, financial problems) determined when symptoms were at their "worst". Several AYA described difficult transitions (e.g. starting college) as stressors that increased depressive symptoms, and practitioners and AYA in LMIC noted the role of previous or ongoing adverse experiences and traumatic events. To cope, AYA reported using distraction (e.g. "keeping busy"), high energy activities (e.g. working out), or engaging in enjoyable activities (e.g. music). Females and AYA 
from the US and UK also talked to others for support, but the majority of AYA had not sought professional counselling.

Practitioners reported using PS for prevention and treatment. Prevention typically involved a single PS session focused on immediate stressors; treatment typically involved 1-2 PS sessions within a larger treatment. Practitioners reported that PS was helpful for specific, solvable problems (e.g. academic problem), but not for "unsolvable" problems (e.g. getting asylum status). AYA consistently mentioned needing to address "the problems" causing the symptoms, including both stressors and beliefs informed by previous experiences (e.g. traumatic events).

Practitioners believed that teaching PS can help reduce stress, increase skills, and shift PS orientation and self-efficacy beliefs but does not meet the needs of all AYA with depression, noting the need to address comorbid symptoms (i.e., trauma, anxiety) as well as underlying cognitive processes.

Both practitioners and AYA noted the importance of individualised pathways to the development and presentation of depression. AYA reported that depression was poorly understood by their peers and society, reporting stigma as a barrier to treatment.

\section{METHODS}

Figure 1: Review approach

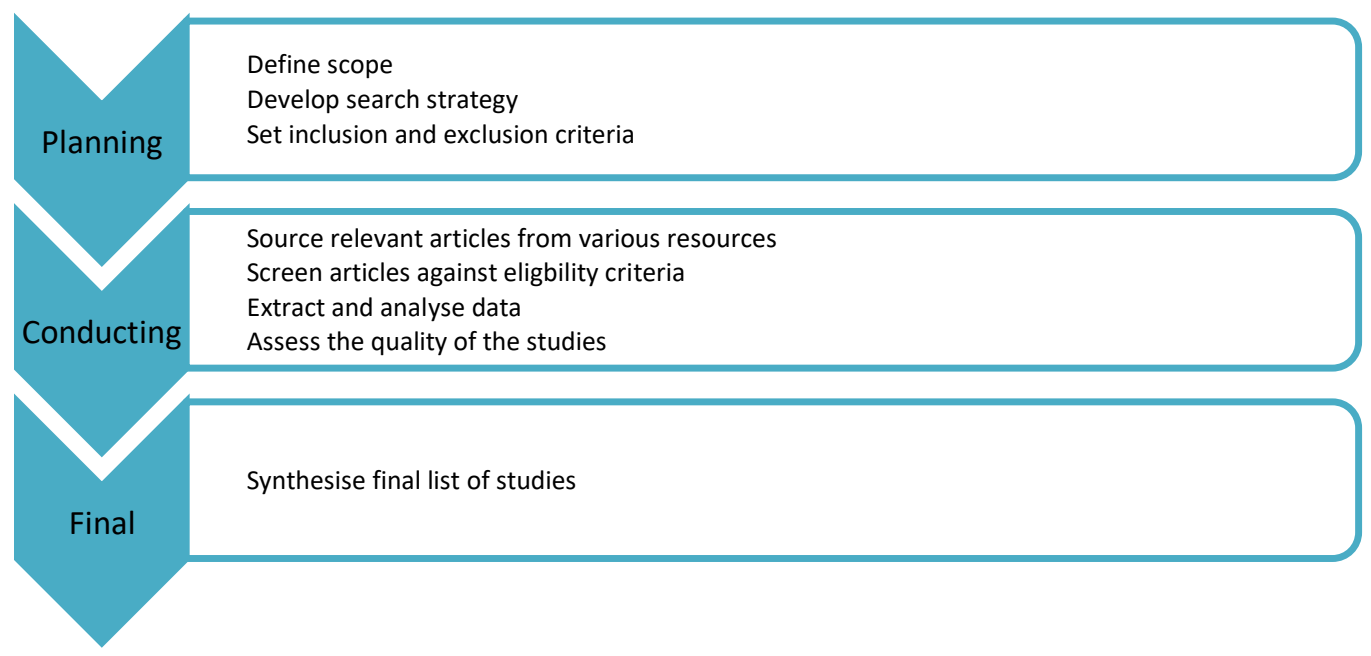

\subsection{Scope of study}


The review was focused by the question: "in which ways and in which contexts and for whom does PS appear to work, and why; and in which ways and in which contexts and for whom do they appear not to work, and why?". The question was further defined in terms of Population, Intervention, Comparison, Outcomes and Study design (PICOS): "Among AYA aged 14-24 (P), is PS including interventions that contain PS (I) effective in decreasing depression symptoms (O)?". Two searches were conducted, the first focused on effectiveness and the second on implementation.

\subsection{Search 1 Strategy: Effectiveness of PS interventions}

Initial searches were conducted using Psyclnfo, Medline, and Cochrane Library with the following search terms: "problem solving", "adolescent", "youth", and "depression" along with filters limiting results to controlled studies looking at effectiveness or exploring mechanisms of effectiveness. Synonyms and derivatives were employed to expand the search. We searched grey literature using Greylit.org and Opengrey.eu, contacted experts in the field and authors of protocols, and searched the reference lists of all included studies,. The search was undertaken on $4^{\text {th }}$ June 2020.

\section{Inclusion and exclusion criteria}

Studies were included if the intervention was described by authors as a PS intervention or including PS, used to treat or prevent depression among AYA aged 13-252 years, with depression outcomes reported. Literature in electronic format published post 2000 were deemed eligible, given the greater relevance of more recent usage of PS.

Studies reporting a mean or median age between 13-25 years were included. There was no exclusion for gender, ethnicity, or country setting; only English language texts were included.

We excluded studies of CBT, IPT, Acceptance and Commitment Therapy, Dialectical Behaviour Therapy, and modified forms of these treatments. These treatments include PS and have been shown to demonstrate small to medium effects for depression (Eckshtain et al, 2020; Stice et al., 2009; Weersing et al., 2017), but the unique contribution of PS cannot be disentangled.

\footnotetext{
${ }^{2}$ Although our primary interest was in 14-24 year olds, we widened our inclusion criterion by a year on either side to expand the number of eligible studies. This enabled us to include three studies that would otherwise have been excluded.
} 
Randomised controlled trials (RCTs), quasi-experimental designs (QEDs), systematic reviews/meta-analyses, pilots, or other studies with clearly defined comparison condition (no treatment, treatment as usual (TAU), or a comparator treatment) were included ${ }^{3}$.

Studies were included if conducted in hospitals, health clinics and community-based clinics; schools, colleges and universities; out-of-home care settings, foster care and other social welfare settings; detention centres and juvenile justice settings; family settings, and disaster and conflict zones.

\section{Study selection}

All citations were entered into Endnote and uploaded to Covidence for screening and review against the inclusion/exclusion criteria. Two reviewers with high inter-rater reliability (98\%) independently screened the title and abstract $(\mathrm{SC}, \mathrm{JM})^{4}$. Full text of articles that met criteria were screened by the two reviewers. Duplicates, irrelevant studies, and studies that did not meet criteria were removed and the reason for exclusion recorded. Discrepancies were resolved by the review team lead (JL).

\section{Data Extraction}

Data were extracted by 2 reviewers (SC, JM) to a form that included: (i) study characteristics (author, publication year, location, design, study aim), (ii) population (age, gender, race/ethnicity, education, family income, depression status), (iii) setting, (iv) intervention description (therapeutic or preventative, whether PS was provided alone or as part of a wider intervention, duration, delivery mode), (v) treatment outcomes (measures used and reported outcomes for depression, suicidality, and PS), (vi) fidelity/implementation outcomes.

RCTs were assessed for quality (i.e., confidence in the study's findings) using the Cochrane Risk of Bias 2.0 tool (Sterne et al., 2019), which includes assessment of potential risk of bias relating to the process of randomisation; deviations from the intended intervention/s; missing data; outcome measurement, and reported results. Risk of bias relating to each domain is estimated using an algorithm, grouped as: Low risk; Some Concerns; or High risk. Two

\footnotetext{
${ }^{3}$ Study protocols, case studies, conference papers, book chapters, opinion pieces, and editorials were excluded.

${ }^{4}$ If reviewers could not retrieve the full text, effort was made to contact the authors.
} 
reviewers (SC, LB) assessed the quality of included studies and discrepancies were resolved by consensus.

Table 1 Individual risk of bias assessments using Cochrane RoB2 tool by domain (1-5) and overall (6) 


\begin{tabular}{|c|c|c|c|c|c|c|}
\hline $\begin{array}{l}\text { Study } \\
\text { (Author } \\
\text { and year) }\end{array}$ & $\begin{array}{l}\text { Domain } 1 . \\
\text { Randomisation } \\
\text { process }\end{array}$ & $\begin{array}{l}\text { Domain } 2 . \\
\text { Deviations } \\
\text { from } \\
\text { intended } \\
\text { interventions }\end{array}$ & $\begin{array}{l}\text { Domain } \\
\text { 3. } \\
\text { Missing } \\
\text { outcome } \\
\text { data }\end{array}$ & $\begin{array}{l}\text { Domain } 4 . \\
\text { Measurement } \\
\text { of outcome }\end{array}$ & $\begin{array}{l}\text { Domain } \\
5 . \\
\text { Selection } \\
\text { of the } \\
\text { reported } \\
\text { result }\end{array}$ & $\begin{array}{l}\text { Domain } \\
6 . \\
\text { Overall } \\
\text { risk of } \\
\text { bias }\end{array}$ \\
\hline Bird 2018 & Low risk of bias & $\begin{array}{l}\text { Some } \\
\text { concerns }\end{array}$ & $\begin{array}{l}\text { Some } \\
\text { concerns }\end{array}$ & $\begin{array}{l}\text { Some } \\
\text { concerns }\end{array}$ & $\begin{array}{l}\text { Some } \\
\text { concerns }\end{array}$ & $\begin{array}{l}\text { Some } \\
\text { concerns }\end{array}$ \\
\hline $\begin{array}{l}\text { Brugha } \\
2000\end{array}$ & Low risk of bias & $\begin{array}{l}\text { Some } \\
\text { concerns }\end{array}$ & $\begin{array}{l}\text { Low risk } \\
\text { of bias }\end{array}$ & $\begin{array}{l}\text { Some } \\
\text { concerns }\end{array}$ & $\begin{array}{l}\text { Some } \\
\text { concerns }\end{array}$ & $\begin{array}{l}\text { Some } \\
\text { concerns }\end{array}$ \\
\hline $\begin{array}{l}\text { Chibanda } \\
2014\end{array}$ & Some concerns & $\begin{array}{l}\text { Some } \\
\text { concerns }\end{array}$ & $\begin{array}{l}\text { Some } \\
\text { concerns }\end{array}$ & $\begin{array}{l}\text { High risk of } \\
\text { bias }\end{array}$ & $\begin{array}{l}\text { Some } \\
\text { concerns }\end{array}$ & $\begin{array}{l}\text { Some } \\
\text { concerns }\end{array}$ \\
\hline $\begin{array}{l}\text { Dietz } \\
2014\end{array}$ & Some concerns & $\begin{array}{l}\text { Some } \\
\text { concerns }\end{array}$ & $\begin{array}{l}\text { Low risk } \\
\text { of bias }\end{array}$ & $\begin{array}{l}\text { Low risk of } \\
\text { bias }\end{array}$ & $\begin{array}{l}\text { Some } \\
\text { concerns }\end{array}$ & $\begin{array}{l}\text { Some } \\
\text { concerns }\end{array}$ \\
\hline $\begin{array}{l}\text { Eskin } \\
2008 \\
\end{array}$ & Some concerns & $\begin{array}{l}\text { Some } \\
\text { concerns }\end{array}$ & $\begin{array}{l}\text { Some } \\
\text { concerns }\end{array}$ & $\begin{array}{l}\text { Some } \\
\text { concerns }\end{array}$ & $\begin{array}{l}\text { Some } \\
\text { concerns }\end{array}$ & $\begin{array}{l}\text { Some } \\
\text { concerns }\end{array}$ \\
\hline $\begin{array}{l}\text { Fitzpatrick } \\
2005 \\
\end{array}$ & Some concerns & $\begin{array}{l}\text { Some } \\
\text { concerns }\end{array}$ & $\begin{array}{l}\text { Low risk } \\
\text { of bias }\end{array}$ & $\begin{array}{l}\text { Some } \\
\text { concerns }\end{array}$ & $\begin{array}{l}\text { Some } \\
\text { concerns }\end{array}$ & $\begin{array}{l}\text { Some } \\
\text { concerns }\end{array}$ \\
\hline $\begin{array}{l}\text { Gaffney } \\
2014\end{array}$ & $\begin{array}{l}\text { High risk of } \\
\text { bias }\end{array}$ & $\begin{array}{l}\text { Some } \\
\text { concerns }\end{array}$ & $\begin{array}{l}\text { Low risk } \\
\text { of bias }\end{array}$ & $\begin{array}{l}\text { Some } \\
\text { concerns }\end{array}$ & $\begin{array}{l}\text { Some } \\
\text { concerns }\end{array}$ & $\begin{array}{l}\text { Some } \\
\text { concerns }\end{array}$ \\
\hline $\begin{array}{l}\text { Gureje } \\
2019\end{array}$ & Low risk of bias & $\begin{array}{l}\text { Some } \\
\text { concerns }\end{array}$ & $\begin{array}{l}\text { Low risk } \\
\text { of bias }\end{array}$ & $\begin{array}{l}\text { Low risk of } \\
\text { bias }\end{array}$ & $\begin{array}{l}\text { Some } \\
\text { concerns }\end{array}$ & $\begin{array}{l}\text { Some } \\
\text { concerns }\end{array}$ \\
\hline $\begin{array}{l}\text { Haeffel } \\
2017\end{array}$ & Some concerns & $\begin{array}{l}\text { High risk of } \\
\text { bias }\end{array}$ & $\begin{array}{l}\text { High risk } \\
\text { of bias }\end{array}$ & $\begin{array}{l}\text { High risk of } \\
\text { bias }\end{array}$ & $\begin{array}{l}\text { Some } \\
\text { concerns }\end{array}$ & $\begin{array}{l}\text { High risk } \\
\text { of bias }\end{array}$ \\
\hline $\begin{array}{l}\text { Hallford } \\
2016\end{array}$ & Low risk of bias & $\begin{array}{l}\text { Low risk of } \\
\text { bias }\end{array}$ & $\begin{array}{l}\text { Some } \\
\text { concerns }\end{array}$ & $\begin{array}{l}\text { Some } \\
\text { concerns }\end{array}$ & $\begin{array}{l}\text { Some } \\
\text { concerns }\end{array}$ & $\begin{array}{l}\text { Some } \\
\text { concerns }\end{array}$ \\
\hline $\begin{array}{l}\text { Hoek } \\
2012\end{array}$ & Low risk of bias & $\begin{array}{l}\text { Some } \\
\text { concerns }\end{array}$ & $\begin{array}{l}\text { Low risk } \\
\text { of bias }\end{array}$ & $\begin{array}{l}\text { Some } \\
\text { concerns }\end{array}$ & $\begin{array}{l}\text { Some } \\
\text { concerns }\end{array}$ & $\begin{array}{l}\text { Some } \\
\text { concerns }\end{array}$ \\
\hline $\begin{array}{l}\text { Hood } \\
2018 \\
\end{array}$ & Some concerns & $\begin{array}{l}\text { Some } \\
\text { concerns }\end{array}$ & $\begin{array}{l}\text { Low risk } \\
\text { of bias }\end{array}$ & $\begin{array}{l}\text { Some } \\
\text { concerns }\end{array}$ & $\begin{array}{l}\text { Some } \\
\text { concerns }\end{array}$ & $\begin{array}{l}\text { Some } \\
\text { concerns }\end{array}$ \\
\hline $\begin{array}{l}\text { Kolko } \\
2000\end{array}$ & Low risk of bias & $\begin{array}{l}\text { Some } \\
\text { concerns }\end{array}$ & $\begin{array}{l}\text { Low risk } \\
\text { of bias }\end{array}$ & $\begin{array}{l}\text { High risk of } \\
\text { bias }\end{array}$ & $\begin{array}{l}\text { Some } \\
\text { concerns }\end{array}$ & $\begin{array}{l}\text { Some } \\
\text { concerns }\end{array}$ \\
\hline $\begin{array}{l}\text { Makover } \\
2019\end{array}$ & Some concerns & $\begin{array}{l}\text { Some } \\
\text { concerns }\end{array}$ & $\begin{array}{l}\text { Low risk } \\
\text { of bias }\end{array}$ & $\begin{array}{l}\text { Some } \\
\text { concerns }\end{array}$ & $\begin{array}{l}\text { Some } \\
\text { concerns }\end{array}$ & $\begin{array}{l}\text { Some } \\
\text { concerns }\end{array}$ \\
\hline $\begin{array}{l}\text { Parker } \\
2016\end{array}$ & Low risk of bias & $\begin{array}{l}\text { Some } \\
\text { concerns }\end{array}$ & $\begin{array}{l}\text { Some } \\
\text { concerns }\end{array}$ & $\begin{array}{l}\text { Low risk of } \\
\text { bias }\end{array}$ & $\begin{array}{l}\text { Some } \\
\text { concerns }\end{array}$ & $\begin{array}{l}\text { Some } \\
\text { concerns }\end{array}$ \\
\hline $\begin{array}{l}\text { Xavier } \\
2019\end{array}$ & Low risk of bias & $\begin{array}{l}\text { Low risk of } \\
\text { bias }\end{array}$ & $\begin{array}{l}\text { Low risk } \\
\text { of bias }\end{array}$ & $\begin{array}{l}\text { Low risk of } \\
\text { bias }\end{array}$ & $\begin{array}{l}\text { Low risk } \\
\text { of bias }\end{array}$ & $\begin{array}{l}\text { Low risk } \\
\text { of bias }\end{array}$ \\
\hline
\end{tabular}

Figure 2: Risk of bias summaries $(n=16)$ 


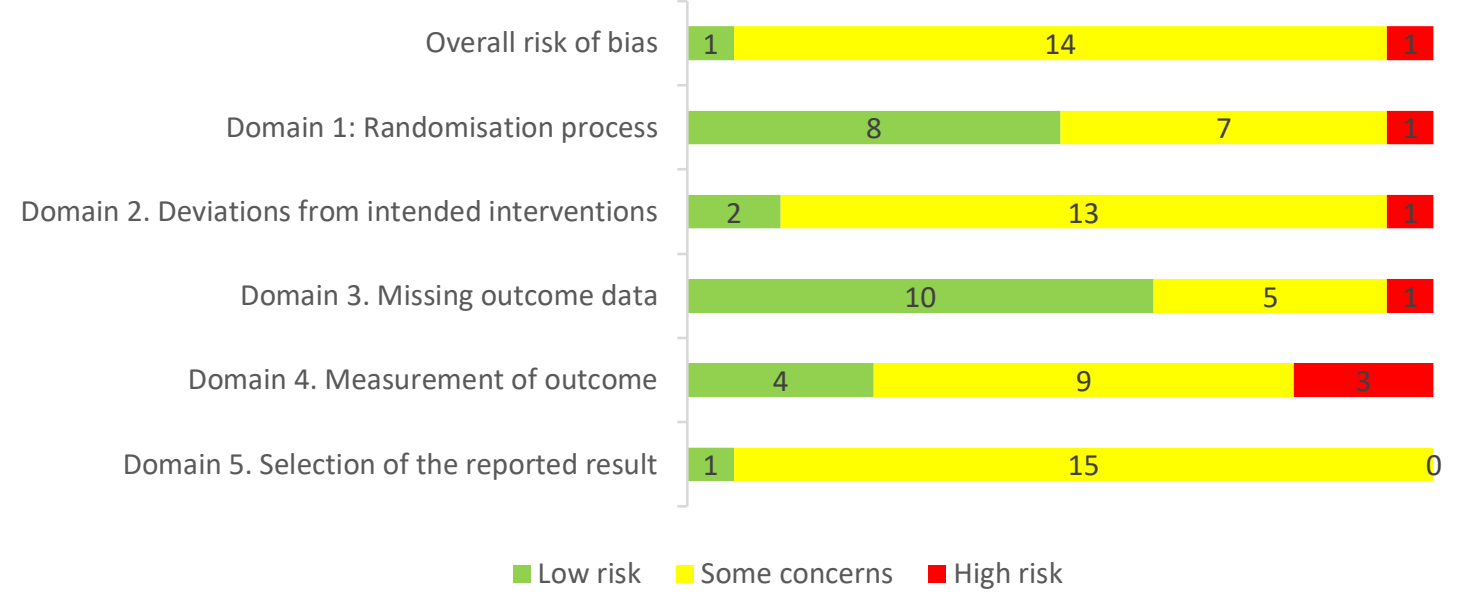

A narrative synthesis was undertaken given the high level of heterogeneity in population, intervention, comparison condition, outcome, and study design.

\subsection{Search 2 strategy - Implementation of PS interventions}

Our second search focused on implementation of PS interventions. The Search 1 strategy was used, but with filters for qualitative and process studies (e.g., "qualitative", "ethnology", "phenomenology", "lived experience", "survey", "focus group", and "process evaluation"). Search 2 was undertaken on 20th July 2020.

Studies were included if they reported on implementation outcomes including feasibility, acceptability, fidelity, and adaptability. Citations were entered into Endnote and Excel for screening and review against inclusion criteria. No risk of bias assessments were conducted. 


\section{RESULTS}

\subsection{Effectiveness of PS interventions}

974 references were identified; 169 were removed as duplicates and 635 excluded during screening. The full text of the remaining 170 was screened. 152 were excluded leaving 18 studies (see PRISMA diagram Figure 3).

\section{Figure 3: PRISMA diagram for Search 1 - effectiveness of PS interventions}

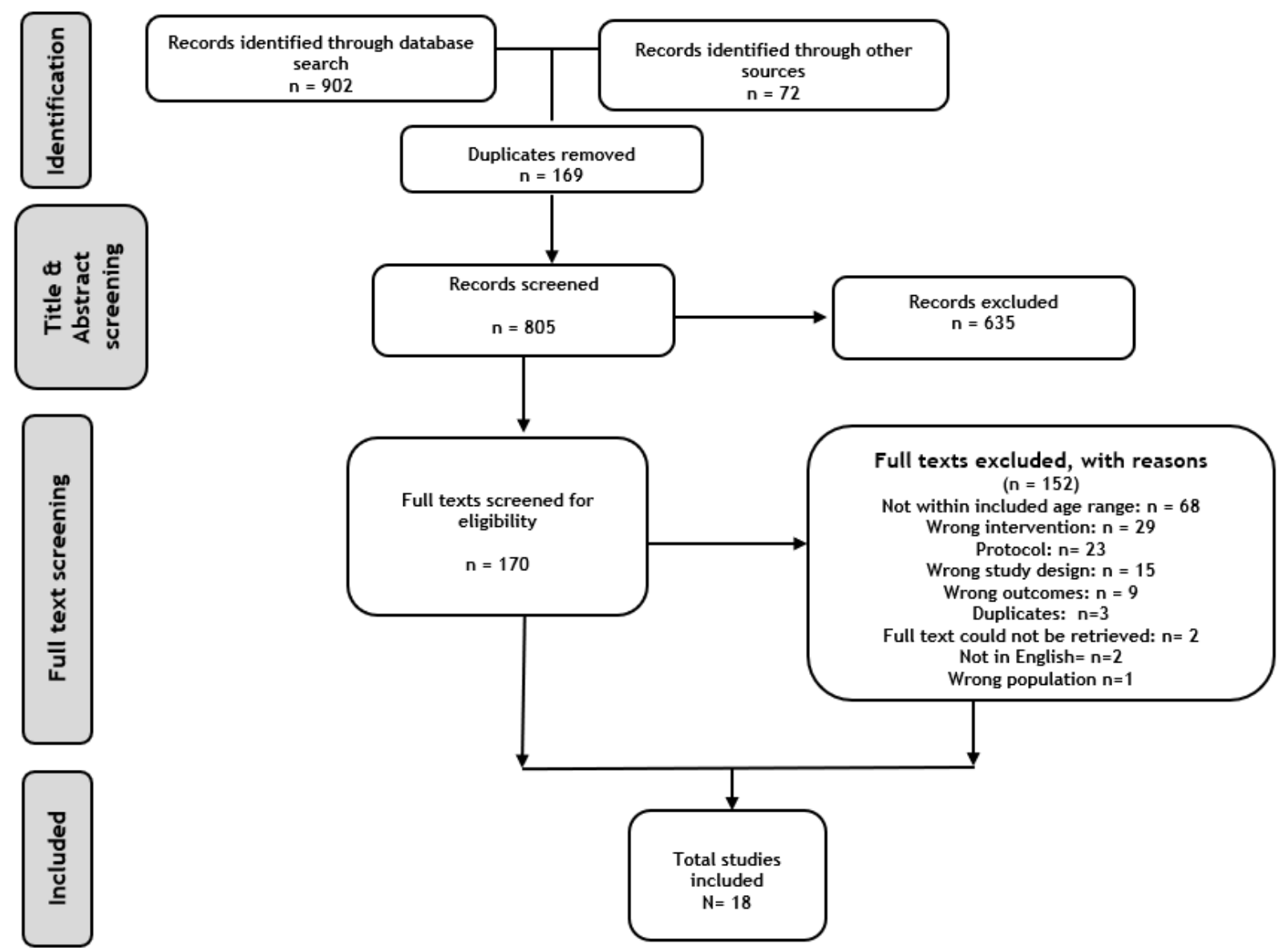

Table 3 presents key characteristics of included studies. Across the 18 studies, 16 interventions were described. 
Table 3: Reviewed studies - effectiveness of PS interventions

\begin{tabular}{|c|c|c|c|c|c|c|c|}
\hline No. & $\begin{array}{l}\text { STUDY } \\
\text { DETAILS }\end{array}$ & $\begin{array}{l}\text { INTERVENTION } \\
\text { PURPOSE }\end{array}$ & INTERVENTION CHARACTERISTICS & $\begin{array}{l}\text { STUDY } \\
\text { DESIGN }\end{array}$ & CONTROL TREATMENT & STUDY POPULATION & $\begin{array}{l}\text { OUTCOMES RELATIVE TO CONTROL } \\
\text { AND MEASURE }\end{array}$ \\
\hline \multicolumn{8}{|c|}{ PROBLEM SOLVING AS STANDALONE TREATMENT } \\
\hline 1 & $\begin{array}{l}\text { Bird et al., } \\
2018 \\
\text { UK }\end{array}$ & $\begin{array}{l}\text { Therapeutic } \\
\text { Problem-related } \\
\text { distress }\end{array}$ & $\begin{array}{l}\text { MYLO: Online, individual problem-solving program based } \\
\text { on PCT principles } \\
\text { Duration could be as long as participants chose (minimum } \\
15 \text { minutes) } \\
\text { Coverage of PST steps unclear }\end{array}$ & $\begin{array}{l}\mathrm{RCT} \\
\mathrm{N}=213\end{array}$ & $\begin{array}{l}\text { ELIZA text-based programme } \\
\text { emulating Rogerian } \\
\text { psychotherapist }\end{array}$ & $\begin{array}{l}\text { University students } \\
\text { No inclusion criteria for depression }\end{array}$ & $\begin{array}{l}\text { No significant change in depression } \\
\text { (DASS-21) } \\
\text { No significant change in problem-solving } \\
\text { (study-developed rating) }\end{array}$ \\
\hline 2 & $\begin{array}{l}\text { Chibanda et } \\
\text { al., } 2014 \\
\text { Zimbabwe }\end{array}$ & $\begin{array}{l}\text { Therapeutic } \\
\text { Postnatal } \\
\text { depression }\end{array}$ & $\begin{array}{l}\text { PST: Group, face-to-face PST intervention } \\
2 \text { sessions per week for } 6 \text { weeks delivered by trained peer } \\
\text { counsellors } \\
\text { Based on Mynors-Wallis (2005) } \\
\text { All/most PST steps covered }\end{array}$ & $\begin{array}{l}\mathrm{RCT} \\
\mathrm{N}=58\end{array}$ & $\begin{array}{l}\text { Amitriptyline } \\
\text { (antidepressant) and peer } \\
\text { education }\end{array}$ & $\begin{array}{l}\text { Women attending primary care clinics } \\
\text { Met criteria for post-partum depression } \\
\text { (DSM-IV) }\end{array}$ & $\begin{array}{l}\text { Significant reduction in postnatal } \\
\text { depression (EPDS) }\end{array}$ \\
\hline 3 & $\begin{array}{l}\text { Eskin et al., } \\
2008 \\
\text { Turkey }\end{array}$ & $\begin{array}{l}\text { Therapeutic } \\
\text { Depression }\end{array}$ & $\begin{array}{l}\text { PST: Individual, face-to-face PST intervention } \\
6 \text { weekly sessions delivered by graduate clinical psychology } \\
\text { students } \\
\text { Based on D'Zurilla and Goldfried (1971) and D'Zurilla and } \\
\text { Nezu (1999) } \\
\text { All/most PST steps covered }\end{array}$ & $\begin{array}{l}\mathrm{RCT} \\
\mathrm{N}=46\end{array}$ & $\overline{W L C}$ & $\begin{array}{l}\text { High school and university students } \\
\text { Diagnosed with MDD (SCIV) }\end{array}$ & $\begin{array}{l}\text { Significant reduction in depression (BDI; } \\
\text { HDRS) and suicide risk (SPS) } \\
\text { No significant change in problem-solving } \\
\text { skills (PSI) }\end{array}$ \\
\hline 4 & $\begin{array}{l}\text { Fitzpatrick } \\
\text { et al., } 2005 \\
\text { US }\end{array}$ & $\begin{array}{l}\text { Therapeutic } \\
\text { Suicidal ideation }\end{array}$ & $\begin{array}{l}\text { Problem-orientation intervention: } 35 \text {-minute video } \\
\text { delivered individually and face-to-face focusing on } \\
\text { problem-solving and coping styles } \\
\text { One session with } 2 \text { modules to be completed } \\
\text { Targeted problem orientation only; covered } 4 \text { steps of PST }\end{array}$ & $\begin{array}{l}\mathrm{RCT} \\
\mathrm{N}=110\end{array}$ & $\begin{array}{l}\text { Single session video covering } \\
\text { health issues inc. diet } \\
\text { exercise and sleep }\end{array}$ & $\begin{array}{l}\text { University students } \\
\text { With suicidal ideation ( } \geq 6 \text { BSS or } \\
\text { endorsing active ideation) }\end{array}$ & $\begin{array}{l}\text { Significant reduction in depression (BDI) } \\
\text { Reduction in suicidal orientation not } \\
\text { sustained (BSS). } \\
\text { No significant change in problem-solving } \\
\text { skills or orientation SPSI-R }\end{array}$ \\
\hline 5 & $\begin{array}{l}\text { Gaffney et } \\
\text { al., } 2014 \\
\text { UK }\end{array}$ & $\begin{array}{l}\text { Therapeutic } \\
\text { Problem-related } \\
\text { distress }\end{array}$ & $\begin{array}{l}\text { MYLO: Online, individual problem-solving program based } \\
\text { on PCT principles } \\
\text { Duration could be as long as participants chose (average } \\
\text { time was } 19.23 \text { minutes) } \\
\text { Coverage of PST steps unclear }\end{array}$ & $\begin{array}{l}\text { Pilot RCT } \\
\mathrm{N}=48\end{array}$ & $\begin{array}{l}\text { ELIZA text-based programme } \\
\text { emulating Rogerian } \\
\text { psychotherapist }\end{array}$ & $\begin{array}{l}\text { University students } \\
\text { No inclusion criteria for depression }\end{array}$ & $\begin{array}{l}\text { No significant change in depression } \\
\text { (DASS-21) } \\
\text { No significant change in problem-solving } \\
\text { (study-developed rating) }\end{array}$ \\
\hline 6 & $\begin{array}{l}\text { Hoek et al., } \\
2012 \\
\text { Netherlands }\end{array}$ & $\begin{array}{l}\text { Preventive } \\
\text { Depression }\end{array}$ & $\begin{array}{l}\text { PST: Individual, online PST intervention } \\
5 \text { weekly sessions completed online with progress } \\
\text { reviewed by mental health care professionals } \\
\text { All/most PST steps covered }\end{array}$ & $\begin{array}{l}\mathrm{RCT} \\
\mathrm{N}=45\end{array}$ & $\overline{W L C}$ & $\begin{array}{l}\text { Recruitment through community and via } \\
\text { parents treated for depression and } \\
\text { anxiety } \\
\text { Self-report of mild or moderate } \\
\text { depression and anxiety. Excluded severe } \\
\text { depression (>40 on CES-D). }\end{array}$ & $\begin{array}{l}\text { No significant change in depression } \\
\text { (CES-D) }\end{array}$ \\
\hline 7 & $\begin{array}{l}\text { Parker et al., } \\
2016 \\
\text { Australia }\end{array}$ & $\begin{array}{l}\text { Therapeutic } \\
\text { Depression }\end{array}$ & $\begin{array}{l}\text { PST: Face-to-face PST intervention. Not specified whether } \\
\text { group or individual. } \\
6 \text { weekly sessions delivered by research psychologists } \\
\text { Based on Mynors-Wallis (2005) } \\
\text { All/most PST steps covered }\end{array}$ & $\begin{array}{l}\text { RCT } \\
\mathrm{N}=176\end{array}$ & $\begin{array}{l}\text { Control treatment based on } \\
\text { general counselling } \\
\text { principles informed by NICE } \\
\text { guidelines for mild to } \\
\text { moderate depression }\end{array}$ & $\begin{array}{l}\text { Young people recruited from youth mental } \\
\text { health services } \\
\text { Elevated symptoms not specific to } \\
\text { depression indicating a mild disorder (K10 } \\
\text { score }>20 \text { ) }\end{array}$ & $\begin{array}{l}\text { No significant change in depression } \\
\text { (BDI) }\end{array}$ \\
\hline 8 & $\begin{array}{l}\text { Tezel \& } \\
\text { Gözüm, } \\
2005 \\
\text { Turkey }\end{array}$ & $\begin{array}{l}\text { Therapeutic } \\
\text { Postnatal } \\
\text { depression }\end{array}$ & $\begin{array}{l}\text { PST: Individual, face-to-face PST intervention } \\
6 \text { weekly sessions delivered by the nurse researcher } \\
\text { All/most PST steps covered }\end{array}$ & $\begin{array}{l}\mathrm{QED} \\
\mathrm{N}=62\end{array}$ & Nursing intervention & $\begin{array}{l}\text { Mothers vising postnatal care service } \\
\text { At risk of post-partum depression (>11 } \\
\text { EPDS) but without major depressive } \\
\text { symptoms }\end{array}$ & $\begin{array}{l}\text { No significant change in depression } \\
\text { (BDI) }\end{array}$ \\
\hline 9 & $\begin{array}{l}\text { Xavier et al., } \\
2019 \\
\text { Brazil }\end{array}$ & $\begin{array}{l}\text { Preventive } \\
\text { Suicidal behaviour }\end{array}$ & $\begin{array}{l}\text { PST: Group, face-to-face PST intervention } \\
5 \text { weekly sessions delivered by experienced psychologist } \\
\text { Based on D'Zurilla and Nezu (2007) and Vazquez et al. } \\
2015) \\
\text { All/most PST steps covered }\end{array}$ & $\begin{array}{l}\mathrm{RCT} \\
\mathrm{N}=100\end{array}$ & TAU & $\begin{array}{l}\text { Poorly performing students recruited from } \\
3 \text { public schools } \\
\text { Met criteria for depression ( } \geq 16 \text { CES-D) } \\
\text { and high risk of suicide (score total } \geq 45 \text { or } \\
\text { critical item score } \geq 3 \text { on ISO-30) but not } \\
\text { major depression }\end{array}$ & $\begin{array}{l}\text { Significant reduction in depression (CES- } \\
\text { D) and suicidal orientation (ISO-30) } \\
\text { Significant improvement in problem- } \\
\text { solving (SPSI-R) }\end{array}$ \\
\hline
\end{tabular}




\begin{tabular}{|c|c|c|c|c|c|c|c|}
\hline No. & $\begin{array}{l}\text { STUDY } \\
\text { DETAILS }\end{array}$ & $\begin{array}{l}\text { TNIERVENTION } \\
\text { PURPOSE }\end{array}$ & TNTERVENTION CHARACTERISTICS & $\begin{array}{l}\text { STUDY } \\
\text { DESIGN }\end{array}$ & CONTROL TREATMENT & STUDYPOPULATION & $\begin{array}{l}\text { OUTCOMES RELATIVE TO CONTROLE AND } \\
\text { MEASURE }\end{array}$ \\
\hline \multicolumn{8}{|c|}{ PROBLEMT-SOLVING AS PART OF WIDER INTERVENTI } \\
\hline 10 & $\begin{array}{l}\text { Brugha et al., } \\
2000 \\
\text { UK }\end{array}$ & $\begin{array}{l}\text { Preventive } \\
\text { Postnatal depression }\end{array}$ & $\begin{array}{l}\text { Preparing for Parenthood: Group, face-to-face cognitive, problem- } \\
\text { solving and social support intervention } \\
6 \text { weekkly sessions delivered by nurses and occupational therapists } \\
\text { Coverage of PST steps unclear }\end{array}$ & $\begin{array}{l}\mathrm{RCT} \\
\mathrm{N}=292\end{array}$ & TAU & $\begin{array}{l}\text { Mothers attending antenatal dinics } \\
\text { Increased risk of post-natal depression (1+ items } \\
\text { on modified GHQ) }\end{array}$ & $\begin{array}{l}\text { No significant change in postnatal depression } \\
\text { (EPDS; GHQ-D) }\end{array}$ \\
\hline 11 & $\begin{array}{l}\text { Dietz et al., } \\
2014 \\
\text { US }\end{array}$ & $\begin{array}{l}\text { Therapeutic } \\
\text { Depression }\end{array}$ & $\begin{array}{l}\text { Systemic-behavioural Family Therapy(SBFT)(familly-based, face-to-- } \\
\text { face) aimed to treat family dysfunction and teach problem-solving } \\
\text { skills to families. Phase } 1 \text { involved } 12-16 \text { weekly sessions, phase } 2 \\
\text { involved 2-4 booster sessions. Delivered by trained therapists } \\
\text { Coverage of PST steps unclear }\end{array}$ & $\begin{array}{l}\mathrm{RCT} \\
\mathrm{N}=63\end{array}$ & CBT or NST & $\begin{array}{l}\text { Patients recruited from } 2 \text { mental health clinics } \\
\text { Met DSM criteria for MDD ( } \geq 13 \text { BDI) }\end{array}$ & $\begin{array}{l}\text { No significant improvement in depression } \\
\text { (DEP-13 and BDI). CBT and SFBT associated } \\
\text { with increased problem solving (video rating), } \\
\text { Problem solving mediated the association } \\
\text { between CBT (but not SFBT) and remission } \\
\text { from depression. }\end{array}$ \\
\hline 12 & $\begin{array}{l}\text { Gureje et al., } \\
2019 \\
\text { Nigeria }\end{array}$ & $\begin{array}{l}\text { Therapeutic } \\
\text { Perinatal depression }\end{array}$ & $\begin{array}{l}\text { Tndividual, face-to-face problem-solving intervention adapted from } \\
\text { PST-PC. } 8 \text { initial weekly sessions, followed by } 4 \text { fortnightly sessions, } \\
\text { third stage with option of pharmacotherapy/specialist referral for } \\
\text { patients with higher EPD scores } \\
\text { Delivered by primary maternal care providers } \\
\text { Coverage of PST steps unclear }\end{array}$ & $\begin{array}{l}\mathrm{RCT} \\
\mathrm{N}=686\end{array}$ & $\begin{array}{l}\text { Enhanced care as usual including } \\
\text { psycho-education and social } \\
\text { support }\end{array}$ & $\begin{array}{l}\text { Mothers attending childcare clinics } \\
\text { Met criteria for major depression ( } 12 \text { EPDS) }\end{array}$ & $\begin{array}{l}\text { Significant reduction in depression symptoms } \\
\text { but not in remission rate c (EPDS). Significant } \\
\text { increase in remission rate for subgroup of } \\
\text { women with more severe baseline depression } \\
\text { compared to control. }\end{array}$ \\
\hline 13 & $\begin{array}{l}\text { Haeffel et al., } \\
2017 \\
\text { US }\end{array}$ & $\begin{array}{l}\text { Therapeutic } \\
\text { Depression }\end{array}$ & $\begin{array}{l}\text { Social Problem-Solving Therapy: Group, face-to-face intervention } \\
\text { designed to increase social problem-solving and social skills } \\
10 \text { sessions deliliered by trained correctional officers } \\
\text { Covered most PST steps except testing solution }\end{array}$ & $\begin{array}{l}\mathrm{RCT} \\
\mathrm{N}=296\end{array}$ & TAU-psychosocial support & $\begin{array}{l}\text { Juvenile detainees in state-run detention centres } \\
\text { No inclusion criteria for depression }\end{array}$ & $\begin{array}{l}\text { No significant change in depression compared } \\
\text { to control (CDI). Significant reduction in } \\
\text { depression for sub-group with higher } \\
\text { intelligence. Significant increase in depression } \\
\text { for participants with lower intelligence. }\end{array}$ \\
\hline 14 & $\begin{array}{l}\text { Hallford\& } \\
\text { Mellor, } 2016 \\
\text { Australia }\end{array}$ & $\begin{array}{l}\text { Therapeutic } \\
\text { Depression }\end{array}$ & $\begin{array}{l}\text { Cognitive Reminiscence Therapy: Individual, face-to-face cognitive } \\
\text { therapy that included brief PST } \\
6 \text { weekkly sessions delivered by registered provisional psychologist } \\
\text { Coverage of PST steps unclear }\end{array}$ & $\begin{array}{l}\mathrm{RCT} \\
\mathrm{N}=26\end{array}$ & Brief evidence-based treatment & $\begin{array}{l}\text { Young people recruited from a community youth } \\
\text { mental health service } \\
\text { At least moderate depression (score } \geq 7 \text { on DASS- } \\
\text { 21) }\end{array}$ & $\begin{array}{l}\text { No significant reduction in depression (DASS- } \\
\text { 21) }\end{array}$ \\
\hline 15 & $\begin{array}{l}\text { Hood et al., } \\
2018 \\
\text { US }\end{array}$ & $\begin{array}{l}\text { Preventive } \\
\text { Diabetes distress }\end{array}$ & $\begin{array}{l}\text { Penn Resillence Program Type 1Dlabetes: Group, face-to-face } \\
\text { resilience enhancing intervention with a focus on diabetes } \\
\text { management. Teaches cognitive-behavioural, social and problem- } \\
\text { solving skills. } 9 \text { bi-weekly sessions delivered by masters-level } \\
\text { clinicians } \\
\text { Coverage of PST steps unclear }\end{array}$ & $\begin{array}{l}\mathrm{RCT} \\
\mathrm{N}=264\end{array}$ & $\begin{array}{l}\text { Diabetes educational } \\
\text { intervention }\end{array}$ & $\begin{array}{l}\text { Patients from diabetes clinics } \\
\text { No inclusion criteria for depression - excluded } \\
\text { with depression diagnosis or treatment }\end{array}$ & $\begin{array}{l}\text { No significant reduction in depression (CDI) } \\
\text { No significant improvement in problem- } \\
\text { solving (SRSI-R) }\end{array}$ \\
\hline 16 & $\begin{array}{l}\text { Kolko et al. } \\
(2000)\end{array}$ & $\begin{array}{l}\text { Therapeutic } \\
\text { Depression }\end{array}$ & $\begin{array}{l}\text { Systemic-behaviouralfamily Therapy (familly-based, face-to-face) } \\
\text { aimed to treat family dysfunction and teach problem-solving skills } \\
\text { to families. Phase } 1 \text { involved } 12 \text {-16 weekly sessions, phase } 2 \\
\text { involved } 2-4 \text { booster sessions. Delivered by trained therapists } \\
\text { Coverage of PST steps unclear }\end{array}$ & $\begin{array}{l}\mathrm{RCT} \\
\mathrm{N}=107\end{array}$ & CBT or NST & $\begin{array}{l}\text { Patients recrulted from } 2 \text { mental health clinics } \\
\text { Met DSM criteria for MDD ( } 213 \mathrm{BDI})\end{array}$ & $\begin{array}{l}\text { No significant reduction in depression (DEP-13 } \\
\text { and BDI) }\end{array}$ \\
\hline 17 & $\begin{array}{l}\text { Makover et } \\
\text { al., } 2019 \\
\text { us }\end{array}$ & $\begin{array}{l}\text { Preventive } \\
\text { Depression }\end{array}$ & $\begin{array}{l}\text { High School Transition Program: Group and individual, face-to-face } \\
\text { intervention designed to increase social and academic problem- } \\
\text { solving skills.12 group sessions followed by } 4 \text { individual booster } \\
\text { sessions delivered by trained mental health counsellors. Combined } \\
\text { depression and transition programmes } \\
\text { Coverage of PST steps unclear }\end{array}$ & $\begin{array}{l}\mathrm{RCT} \\
\mathrm{N}=497\end{array}$ & $\begin{array}{l}\text { Tnterview and clinical follow-up } \\
\text { without active therapy }\end{array}$ & $\begin{array}{l}\text { Middle and high school students } \\
\text { Met criteria for depression ((score } \geq 15 \text { on MFQ) }\end{array}$ & Significant reduction in depression (MFo) \\
\hline 18 & $\begin{array}{l}\text { Noh, } 2018 \\
\text { South Korea }\end{array}$ & $\begin{array}{l}\text { Preventive } \\
\text { Build resilience and } \\
\text { reduce impact of } \\
\text { trauma }\end{array}$ & $\begin{array}{l}\text { Resililence enhancement program: Group, face-to-face intervention } \\
\text { designed to increase resilience with a component on problem- } \\
\text { solving } \\
2 \text { sessions per week for } 4 \text { weeks delivered by the author and a } \\
\text { psychiatric nurse } \\
\text { Coverage of PST steps unclear }\end{array}$ & $\begin{array}{l}Q E D D \\
N=32\end{array}$ & TAU by youth shelters & $\begin{array}{l}\text { Runaway youths from homeless shelters } \\
\text { No inclusion criteria for depression - excluded } \\
\text { young people receiving psychiatric interventions }\end{array}$ & No significant reduction in depression (BDI) \\
\hline \multicolumn{8}{|c|}{ 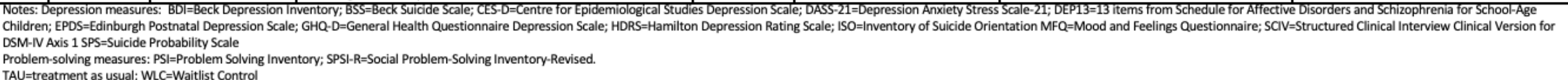 } \\
\hline
\end{tabular}




\subsubsection{Overview of studies}

\section{Intervention characteristics}

Eight interventions focused purely on PS, six covering five to seven steps of PS Therapy (PST) with each step described in detail ${ }^{5}$. Three were adaptations of models proposed by D'Zurilla and Nezu (1999, 2007) and D'Zurilla and Goldfried (1971), two were based on Mynors-Wallis (2005)'s PST guide and one did not specify a model. A problem-orientation video intervention adapted from D'Zurilla and Nezu (1999), focused on the first four steps of PST. The last purely PS intervention was an online intervention adapted from Method of Levels therapy.

Eight interventions used PS as part of a wider intervention (e.g. cognitive therapy, social support and skills, and resilience enhancement). How, and to what extent, PS skills were covered in these interventions was unclear.

Ten were described as therapeutic interventions and six as preventive. Seven studies referred to the intervention having been developed or adapted for young people.

\section{Modality}

Seven interventions were delivered individually, six were group-based, one was family-based, one was mixed, and in one the format of delivery was unclear. Thirteen were delivered face-toface and three online. Dosage ranged from a single session to 16 weekly sessions plus 4 booster sessions: the mode was once per week for 6 weeks $(\mathrm{N}=5)$.

\section{Study settings and populations}

Thirteen studies were conducted in high-income countries (UK, US, Australia, Netherlands, South Korea), three in upper-middle income (Brazil, Turkey), and two in lower-middle income countries (Zimbabwe, Nigeria).

Two studies included participants younger than 13 and three older than 25 . Six studies were conducted on university or high school student populations and four on pregnant or post-partum mothers. The remaining eight used populations from mental health clinics, the community, a diabetes clinic, juvenile detention, and a runaway shelter.

\footnotetext{
${ }^{5}$ Based on the CETA seven steps shown in Box 1 which reflect common steps across model
} 
Twelve studies included participants who met the criteria for a depressive or suicidal disorder, (two of these excluded severe depression). Six studies did not use depression symptoms in the inclusion criteria (one of these excluded depression).

Several studies excluded other significant mental health conditions.

\section{Study designs}

Sixteen studies were RCTs; two were QEDs. Seven had TAU or wait-list control (WLC) comparator groups, 11 used active control groups (e.g., alternative treatment). Eight studies described fidelity measures. Sample size ranged from 26 to 686 and was under 63 in eight studies.

Risk of bias concerns were moderate and a fair degree of confidence in the validity of study findings is warranted. The majority of studies (88\%) were assessed as 'some concerns' $(\mathrm{N}=14)$, one study $\left(9^{6}\right)$ was 'low risk', and one (13) 'high risk'. The most frequent areas of concern was selection of the reported result $(n=15$, mostly due to inadequate reporting of a priori analytic plans); deviations from the intended intervention $(\mathrm{N}=14)$, mostly related to insufficient information about intention-to-treat analyses); and measurement of outcome ( $\mathrm{N}=12)$.

\section{Outcome measures}

Seven interventions targeted depression, four post/perinatal depression, two suicidal ideation, one 'problem-related distress', one 'diabetes distress', and one resilience. Those targeting post/perinatal depression used the Edinburgh Postnatal Depression Scale as the outcome measure. Of the others, six used the Beck Depression Inventory, two the Children's Depression Inventory, three the Depression Anxiety Stress Scale-21, two the Centre for Epidemiologic Studies Depression Scale, one the Short Mood and Feelings Questionnaire, one the Hamilton Depression Rating Scale, and one the depression subscale on the Schedule for Affective Disorders and Schizophrenia for School-Age Children.

Only seven studies measured PS skills or orientation outcomes. Three used the Social Problem-Solving Inventory-Revised, one the Problem Solving Inventory, two measured the

\footnotetext{
${ }^{6}$ Numbers refer to the numbered studies in Table 3
} 
extent to which the nominated problem had been resolved, and one observed PS in video-taped interactions.

\subsubsection{Outcomes}

The mixed findings regarding the effectiveness of PS for depression may depend on whether substantial depression is observed among study participants prior to treatment. Outcomes are therefore grouped by outcome, severity of clinical features, and comparator treatment. Within these groupings studies with lower risk of bias (RCTs) are presented first.

\section{Reduction in depression symptoms}

\section{AYA meeting criteria for depressive disorder - compared to no treatment}

A small but high quality (low-risk of bias) study (9) focused on preventing suicidal risk among school students in Brazil (RCT, $\mathrm{N}=100$, low risk of bias), where treatment covered all/most PST steps, found a significant, moderate reduction in depression symptoms for the treatment group post-intervention, fairly well-maintained at 1-month, 3-month and 6-month follow-up.

A study (3) of an intervention for depression and suicidal proneness among high school and university students in Turkey (RCT, $\mathrm{N}=46$, moderate risk of bias), covering all/most PST steps, found large effect sizes on post-treatment depression scores for intervention participants posttreatment compared with WLC; improvements were maintained at 12-months follow-up.

The High School Transition Program (17) in the US (RCT, N=497, moderate risk of bias) aimed to prevent depression, anxiety, and school problems in youth at the transition to high school; coverage of PST steps was unclear. There was a small reduction in the percentage of intervention students with clinical depression compared to the control group at the 18-month study period, however this difference was marginally significant.

However, an online PS intervention delivered to young people in the Netherlands (6) to prevent depression (RCT, $\mathrm{N}=45$, moderate risk of bias), covering all/most PST steps, found no significant difference in depression level at 3 and 5 weeks and 4 months post-treatment. 
AYA meeting criteria for depressive disorder - compared to active control

A problem-orientation intervention covering four PST steps and involving a single session video for US university students (4) (RCT, $n=110$, moderate risk of bias), compared with a video covering other health issues, resulted in a moderate reduction in depression post-treatment. While an overall reduction was seen compared to baseline, depression scores did not continue to decline at 2 weeks and 1 month follow up.

Cognitive Reminiscence Therapy which involved recollection of past PS experiences and drew on PS techniques (14) used for 12-25 year olds in community mental health services in Australia (a small, $\mathrm{RCT}(\mathrm{N}=26)$ with moderate risk of bias) did not reduce depression symptoms compared with a brief evidence-based treatment at one or three months follow-up.

Two US studies $(11,16)$ used a three-arm trial to compare Systemic-behavioural Family Therapy (SBFT) with elements of PS, to CBT and individual Non-directive Supportive therapy (NST) (RCT, $\mathrm{N}=107$, moderate risk of bias). There were no significant differences in depression between CBT, SBFT and NST at two-year follow-up

AYA meeting criteria for perinatal depression - compared to no treatment A preventive manualised programme for postnatal depression that included PS approaches (coverage of PST steps unclear) tested in the UK (10), found no significant differences in depression scores at three months post-partum ( $R C T, N=292$, moderate risk of bias).

AYA meeting criteria for perinatal depression - compared to active control Three further studies of perinatal depression used an active control group. An intervention among women in Zimbabwe (2) (RCT, N=58, moderate risk of bias) covering all/most PST steps and found a larger decrease in Edinburgh Postnatal Depression Scale score for the intervention group compared to control (who received the antidepressant amitriptyline and peer education) at 6 weeks post-treatment.

A PS intervention tested in maternal and child clinics in Nigeria (coverage of PST steps unclear) (12) RCT ( $\mathrm{N}=686$, moderate risk of bias) compared with enhanced TAU involving psychosocial and social support, found no significant difference in the proportion of women who recovered from depression at six months post-partum, although there was a small difference in depression scores in favour of PS averaged across the 3, 6, 9, and 12 month follow-up points. 
A study in Turkey (8) used a non-equivalent control group design to test a nursing intervention against a PS control intervention (covering all/most PST steps). This was a QED $(\mathrm{N}=62)$. Both groups showed a reduction in depression, but the nursing care intervention demonstrated a larger decrease post-intervention than the PS control intervention.

AYA without criteria for depressive disorder - compared to non-active control A study of a social PS intervention (13) (which appeared to cover all PS steps with the possible exception of testing strategies) among juveniles in state-run detention centres in the US found no impacts ( $\mathrm{RCT}, \mathrm{N}=296$, high risk of bias).

A QED ( $N=32$ ) was used to test the effectiveness of a resilience enhancement and prevention intervention for runaway youth in South Korea (18) (coverage of PST steps unclear). There was a moderate decrease in depression for the intervention group compared with the control group during the one-month intervention period, but the difference was not sustained at one-month follow-up (BDI).

AYA without criteria for depressive disorder - compared to active control A 2x2 factorial RCT (7) ( $\mathrm{N}=176$, moderate risk of bias) tested PST (covering all/most PST steps) among youth mental health service users with mild mental disorder in Australia was not superior to supportive counselling at two weeks post-treatment.

An adaptation of the Penn Resilience Program for young people with diabetes (15) (RCT, $\mathrm{N}=264$, moderate risk of bias; coverage of PST steps unclear) showed a moderate reduction in diabetes distress but not depression at 4, 8, 12 and 16 months follow-up compared to a diabetes education intervention.

Similarly, no differences were found between groups at two weeks follow-up when the online Manage Your Life Online (coverage of PST steps unclear) was compared with an online programme emulating Rogerian psychotherapy for UK university students with problem-related distress (1) ( $R C T, N=213$, moderate risk of bias); (5) ( $R C T, N=48$, moderate risk of bias). 


\section{Reduction in suicidality}

Three studies measured reduction in suicidality. A preventive treatment (9) found a large reduction in suicidal orientation in the PS group compared to control post-treatment; while suicidal ideation scores were inconsistent at 1-,3- and 6- month follow-up they maintained an overall lower score. In a PST intervention (3), post-treatment suicide risk scores were lower than pre-treatment for the PST group, but unchanged for the control group. An online treatment (4) found a moderate decline in ideation for the intervention group at post-treatment compared to control, not sustained at one-month follow-up.

\subsubsection{Mediators and moderators}

PS abilities and orientation

Seven studies measured PS skills or effectiveness. In two $(3,4)$, despite the interventions reducing depression, there was no change in PS abilities. One (9) found that change in global and functional PS skills mediated the relationship between the intervention group and change in suicidal orientation, but this was not assessed for depression. Three other studies $(1,5,15)$ found no change in depression symptoms or PS skills. Finally, CBT and SBFT (11) led to significant increases in PS behaviour and PS was associated with higher rates of remission across treatments, but did not moderate the relationship between SBFT and remission status.

Other pathways, mediators, and moderators

A high-intensity intervention for perinatal depression in Nigeria had no treatment effect on the whole sample but was significantly effective for participants who had more severe depression at baseline (12).

A PS intervention among juvenile detainees in the US (13) was effective at reducing depression for participants with higher levels of fluid intelligence, but symptoms increased for those with lower levels, the authors suggest because they may have been less able to cope with exploring negative emotions and to apply the skills learned.

\subsection{Implementation of PS interventions}

The search identified 480 references. 41 further were identified through other sources. Three duplicates were removed and 454 excluded in screening. Full text screening of the remaining 64 identified only one eligible study (see Figure 4 for PRISMA diagram). 
Figure 4: PRISMA diagram for Search 2 - implementation studies

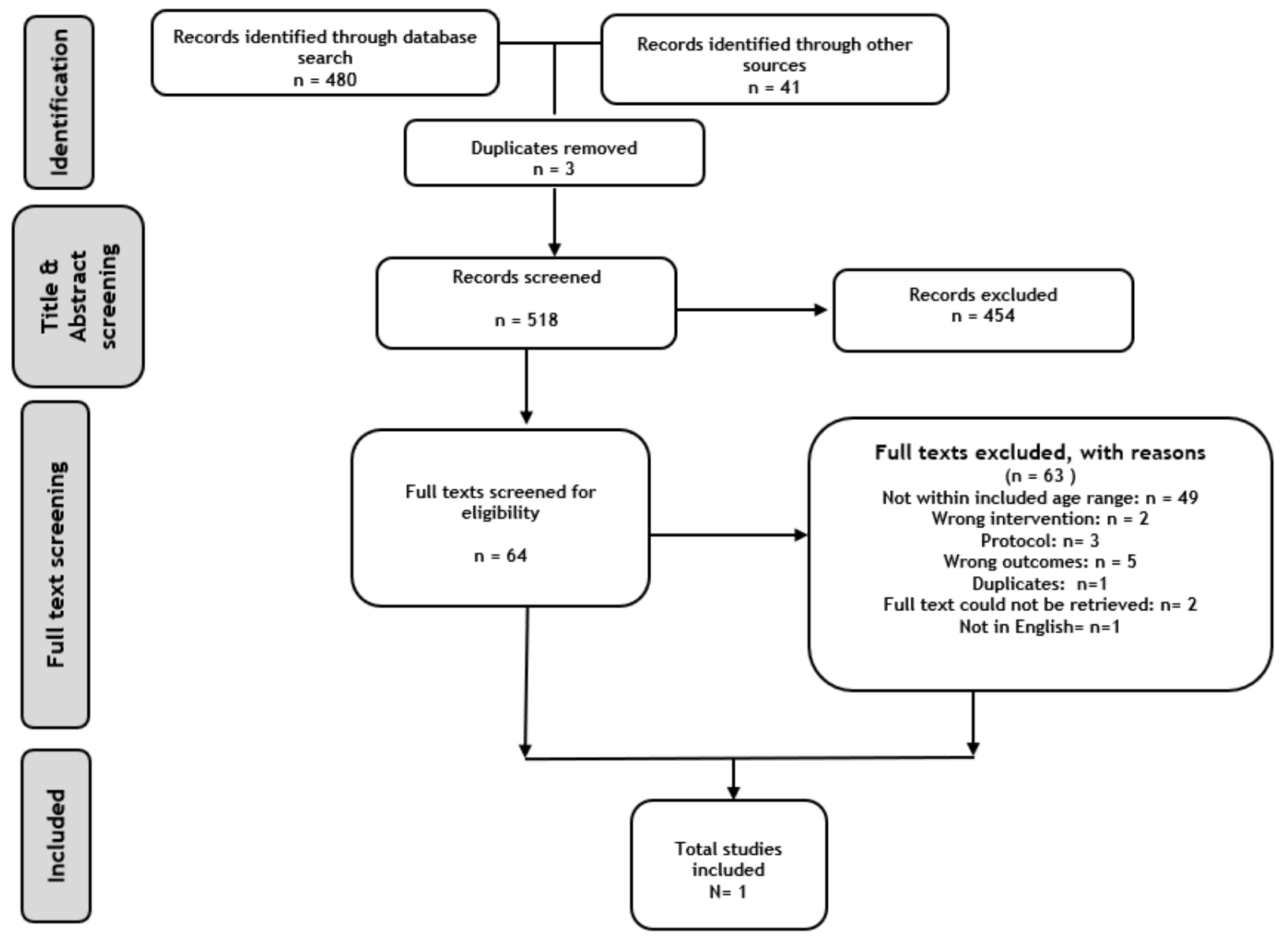

The study aimed to examine the feasibility of adapting a PST intervention for low-income mothers with postnatal depression recruited from Healthy Start clinics in the US (Sampson et al., 2016), consisting of 8 to 16 sessions delivered at home by caseworkers.

The $93 \%$ retention rate, $100 \%$ completion of homework rate and high satisfaction among caseworkers indicated feasibility. Implementation challenges included high burden on caseworkers and fidelity not assessed due to caseworker concerns about video-recording.

There is further evidence in the effectiveness studies reviewed. Where fidelity was monitored (9, $11,12,13,14,16,17)$, high levels were reported. However, studies identified challenges including attrition $(1,4,6,10)$; challenges with operationalising online programmes $(1,6)$; and practitioner skills (10). 


\section{STUDY LIMITATIONS}

This is the first review of PS focused on AYA, and includes PST and multi-component interventions. We excluded studies that included four treatments known to be effective in treating depression among AYA (e.g., CBT), where the unique contribution of PS to clinical outcome could not be disentangled.

We relied on authors' reporting to determine if PS was included: details about operationalisation of PS were often scant. Little evidence addressing the fit, feasibility, or acceptability of PS interventions was found, reflecting a limited focus on implementation. We included only Englishlanguage texts: relevant studies in other languages may exist, though our post-2000 inclusion criteria may limit this potential bias. Finally, the heterogeneity of study populations, problem severity, comparison conditions, outcome measures, and study designs along with a relatively small number of included studies limits confidence in what we can say about implementation and treatment outcomes.

\section{DISCUSSION}

This review has examined the evidence on the effectiveness of PS in the treatment or prevention of depression among 13-25 year olds. We found 18 studies involving 16 interventions. We sought to determine in what way, in which contexts, and for whom PS appears to work in addressing depression. Results were mixed in terms of reducing depression and suicidality. When compared with no treatment, PS were sometimes effective even in highly controlled studies with AYA. Yet, when compared with active control among AYA with at least mild depression, PS was often no more effective than other approaches. As a preventive effort, PS appears fairly limited when depression is not present. Beyond these broad conclusions, it is difficult to discern differences between effective and non-effective studies. Studies varied in terms of location (LMIC and high income contexts); modality and dosage (from a single session video-based intervention to greater frequency sessions; individual and group delivery; online and in-person); and interventions delivered at school, university, and youth mental health services setting. A broad conclusion might be that PS can be delivered effectively in a range of different contexts and forms, is acceptable to a range of populations, and can be tailored to address different types of problems. 
However, it is also clear that many efforts did not succeed, although the scale of impact is broadly in line with the small to moderate effectiveness of other treatments for youth depression (Weisz et al 2019). There are possible explanations for this. As noted, the scope for effectiveness for mild (or no) depressive symptoms may be very limited. It may be unrealistic to expect a stand-alone PS intervention (as eight of the studies were) to decrease depression symptoms especially if comorbid mental health difficulties exist, the problems experienced are not easily resolved, or an equally viable intervention is offered. There was little information about comorbidity in most of the studies and no analysis of whether it influenced outcomes.

Our review also highlights shortcomings in study design, methods, and reporting. Studies varied in how well PS was operationalised. Low dosage is consistent with usage described in informal conversations with practitioners, but may be insufficient for effectiveness. Only six treatments covered all or most PST steps. Fidelity was monitored in only half the studies despite evidence that monitoring implementation improves effectiveness (Durlak \& Dupre, 2009). There were references to implementation difficulties including attrition, challenges in operationalizing online interventions, and skills of those delivering. More information about implementation is essential to understand the potential of PS for wider dissemination.

The small sample size of many studies may mean there were effects that were not found, and there may have been longer-term effects in the preventative studies not captured by short follow-up periods.

Our review found limited evidence about PS skills as mediator or moderator of depression. Few studies measured improvements in PS skills, fewer still found interventions effective here. The absence of evidence for PS abilities as a pathway is puzzling. It may be that specific aspects of PS behaviours and processes (such as problem orientation (Becker-Weidman et al., 2010) are relevant. Our interviews with AYA and with practitioners point to bi-directional and possibly complex relationships between PS and depression, which may weaken the association between PS and depression outcomes. Alternatively, there may be a mechanism other than PS skills through which PS interventions have an effect on depression.

Overall our review points to a need for continued innovation in treatment to improve the operationalising and testing of PS. It also highlights the need for study methods that allow us to 
understand the specific effects of PS, and that measure the frequency, dosage and timing of PS to understand what is effective for whom and in what contexts. 


\section{REFERENCES}

\section{Reviewed studies}

Bird, T., Mansell, W., Wright, J., Gaffney, H., \& Tai, S. (2018). Manage your life online: A webbased randomized controlled trial evaluating the effectiveness of a problem-solving intervention in a student sample. Behavioural and Cognitive Psychotherapy, 46(5), 570-582.

Brugha, T. S., Wheatley, S., Taub, N. A., Culverwell, A., Friedman, T., Kirwan, P., Jones, D. R., \& Shapiro, D. A. (2000). Pragmatic randomized trial of antenatal intervention to prevent postnatal depression by reducing psychosocial risk factors. Psychological Medicine, 30, 1273-1281.

Chibanda, D., Shetty, A. K., Tshimanga, M., Woelk, G., Stranix-Chibanda, L., \& Rusakaniko, S. (2014). Group problem-solving therapy for postnatal depression among HIV-positive and HIVnegative mothers in Zimbabwe. Journal of the International Association of Providers of AIDS Care, 13(4), 335-341.

Dietz, L. J., Marshal, M. P., Burton, C. M., Brudge, J. A., Birmaher, B., Kolko, D., Duffy, J. N., \& Brent, D. A. (2014). Social problem solving among depressed adolescents is enhanced by structured psychotherapies. Journal of Consulting and Clinical Psychology, 82(2), 202-211.

Eskin, M., Ertekin, K., \& Demir, H. (2008). Efficacy of a problem-solving therapy for depression and suicide potential in adolescents and young adults. Cognitive Therapy and Research, 32, 227-245.

Fitzpatrick, K. K., Witte, T. K., \& Schmidt, N. B. (2005). Randomized controlled trial of a brief problem-orientation intervention for suicidal ideation. Behavior Therapy, 36(4), 323-333.

Gaffney, H., Mansell, W., Edwards, R., \& Wright, J. (2014). Manage Your Life Online (MYLO): A pilot trial of a conversational computer-based intervention for problem solving in a student sample. Behavioural and cognitive psychotherapy, 42(6), 731.

Gureje, O., Oladeji, B. D., Montgomery, A. A., Araya, R., Bello, T., Chisholm, D., ... \& Tan, W. (2019). High-versus low-intensity interventions for perinatal depression delivered by nonspecialist primary maternal care providers in Nigeria: Cluster randomised controlled trial (the EXPONATE trial). British Journal of Psychiatry, 215(3).

Haeffel, G. J., Hein, S., Square, A., Macomber, D., Lee, M., Chapman, J., \& Grigorenko, E. L. (2017). Evaluating a social problem solving intervention for juvenile detainees: Depressive outcomes and moderators of effectiveness. Development and psychopathology, 29(3), 10351042.

Hallford, D. J., \& Mellor, D. (2016). Autobiographical Memory-Based Intervention for Depressive Symptoms in Young Adults. Psychotherapy and psychosomatics, 85(4), 246-249. 
Hoek, W., Schuurmans, J., Koot, H. M., \& Cuijpers, P. (2012). Effects of Internet-based guided self-help problem-solving therapy for adolescents with depression and anxiety: A randomized controlled trial. PLoS One, 7(8), e43485.

Hood, K. K., Iturralde, E., Rausch, J., \& Weissberg-Benchell, J. (2018). Preventing diabetes distress in adolescents with type 1 diabetes: Results 1 year after participation in the STePS program. Diabetes Care, 41(8), 1623-1630.

Kolko, D. J., Brent, D. A., Baugher, M., Bridge, J., \& Birmaher, B. (2000). Cognitive and family therapies for adolescent depression: Treatment specificity, mediation, and moderation. Journal of Consulting and Clinical Psychology, 68(4), 603-614.

Makover, H., Adrian, M., Wilks, C., Read, K., Vander Stoep, A., \& McCauley, E. (2019). Indicated Prevention for Depression at the Transition to High School: Outcomes for Depression and Anxiety. Prevention Science, 20(4), 499-509.

Noh, D. (2018). The Effect of a Resilience Enhancement Programme for Female Runaway Youths: A Quasi-Experimental Study. Issues in Mental Health Nursing, 39(9), 764-772.

Parker, A. G., Hetrick, S. E., Jorm, A. F., Mackinnon, A. J., McGorry, P. D., Yung, A. R., ... \& Purcell, R. (2016). The effectiveness of simple psychological and physical activity interventions for high prevalence mental health problems in young people: a factorial randomised controlled trial. Journal of Affective Disorders, 196, 200-209.

Tezel, A., \& Gözüm, S. (2006). Comparison of effects of nursing care to problem solving training on levels of depressive symptoms in post partum women. Patient Education and Counseling, 63(1-2), 64-73.

Xavier, A., Otero, P., Blanco, V., \& Vázquez, F. L. (2019). Efficacy of a problem-solving intervention for the indicated prevention of suicidal risk in young Brazilians: Randomized controlled trial. Suicide and Life-Threatening Behavior, 49(6), 1746-1761.

\section{Other references}

Angold, A., \& Costello, E. J. (2001). The epidemiology of depression in children and adolescents. In I. M. Goodyer (Ed.), Cambridge child and adolescent psychiatry. The depressed child and adolescent (p. 143-178). Cambridge University Press.

Avenevoli, S., Knight, E., Kessler, R. C., \& Merikangas, K. R. (2008). Epidemiology of depression in children and adolescents. In J. R. Z. Abela \& B. L. Hankin (Eds.), Handbook of depression in children and adolescents (p. 6-32). The Guilford Press.

Becker-Weidman, E.G., Jacobs, R.H., Reinecke, M.A., Silva, S.G. \& March, J.S. (2010). Social problem-solving among adolescents treated for depression. Behaviour Research and Therapy 48, 11-18

Bell, A. C., \& D'Zurilla, T. J. (2009). Problem-solving therapy for depression: A meta-analysis Clinical Psychology Review, 29, 348-353. 
Biggam, F. H., \& Power, K. G. (2002). A controlled, problem-solving, group-based intervention with vulnerable incarcerated young offenders. International Journal of Offender Therapy and Comparative Criminology, 46, 678-698.

Boustani, M.M., Frazier, S.L., Becker, K.D., Bechor, M., Dinizulu, S.M., Hedemann, E.R., Ogle, R.R., \& Pasalich, D.S. (2015). Common Elements of Adolescent Prevention Programs:

Minimizing Burden While Maximising Reach. Administration Policy in Mental Health, 42(2), 209219.

Bridge, J. A., Greenhouse, J.B., Weldon, A.H., Campo, J.V., \& Kelleher, K.J. (2008). Suicide Trends Among Youths Aged 10 to 19 Years in the United States, 1996-2005. JAMA, 300(9), 1025-1026.

Chang, E.C. (2002). Predicting suicide ideation in an adolescent population: examining the role of social problem solving as a moderator and a mediator. Personality and Individual Differences, 32 1279-1291

Chibanda, D., Mesu, P., Kajawu, L., Cowan, F., Araya, R., \& Abas, M. A. (2011). Problemsolving therapy for depression and common mental disorders in Zimbabwe: Piloting a taskshifting primary mental health care intervention in a population with a high prevalence of people living with HIV. BMC public Health, 11(1), 828-838.

Chorpita, B.F., \& Daleiden, E.L. (2009). Mapping evidence-based treatments for children and adolescents: Application of the distillation and matching model to 615 treatments from 322 randomized trials. Journal of Consulting and Clinical Psychology, 77(3), 566-579.

D'Zurilla, T. J., \& Nezu, A. M. (1999). Problem-solving therapy: A social competence approach to clinical intervention (2nd ed.). New York: Springer.

D'Zurilla, T.J., \& Nezu, A.M. (2007). Problem-solving therapy: A positive approach to clinical intervention ( $3^{\text {rd }}$ edition) New York: Spring Publishing Company.

D'Zurilla, T. J., \& Goldfried, M. R. (1971). Problem solving and behavior modification. Journal of Abnormal Psychology, 78(1), 107.

Dietz, L. J., Silk, J., \& Amole, M. (2019). Depressive disorders. Eds T. H. Ollendick, S. W. White, \& B. A. White. The Oxford Handbook of Clinical Child and Adolescent Psychology (pp. 280-297). Oxford University Press, New York, NY.

Durlak, J.A. \& DuPre, E.P. (2009) Implementation Matters: A Review of Research on the Influence of Implementation on Program Outcomes and the Factors Affecting Implementation. American Journal of Community Psychology, 41:327-350.

Frye, A.A. \& Goodman, S.H. (2000) Which Social Problem-Solving Components Buffer Depression in Adolescent Girls? Cognitive Therapy and Research 24(6) 637-650

Higgins, J.P.T., Sterne, J.A.C., Savović, J., Page, M.J., Hróbjartsson, A.,Boutron, I., ... Eldridge, S. (2016). A revised tool for assessing risk of bias in randomized trials. In: Chandler J., 
McKenzie J., Boutron I., Welch V. (Eds.), Cochrane Methods. Cochrane Database of Systematic Reviews, Issue 10 (Suppl 1).

Hogue, A., Bobek, M., Dauber, S., Henderson, C. E., McLeod, B. D., \& Southam-Gerow, M. A. (2017). Distilling the core elements of family therapy for adolescent substance use: Conceptual and empirical solutions. Journal of Child and Adolescent Substance Abuse, 26(6), 437-453.

Johnsen T. J., \& Friborg, O. (2016). The effects of cognitive behavioral therapy as an antidepressive treatment is falling: A meta-analysis [published correction appears in Psychol Bull Mar;142(3):290]. Psychological Bulletin, 141(4),747-768.

Kovacs, M., Feinberg, T.L., Crouse-Novak, M.A., Paulauskas, S.L., \& Finkelstein, R. (1984). Depressive Disorders in Childhood: A Longitudinal Prospective Study of Characteristics and Recovery. Archives of General Psychiatry, 41(3), 229-237.

Lewinsohn, P.M., \& Clarke, G.N. (1999). Psychosocial treatments for adolescent depression. Clinical Psychology Review, 19(3), 329-342.

Lewinsohn, P. M., Rohde, P., Seeley, J. R., \& Fisher, S. A. (1993). Age-cohort changes in the lifetime occurrence of depression and other mental disorders. Journal of Abnormal Psychology, $102,110-120$.

Malouf, J.M., Thorsteinsson, E.B., \& Schutte, N.S. (2006). The efficacy of problem solving therapy in reducing mental and physical health problems: A meta-analysis. Clinical Psychology Review, 27, 46-57.

Merikangas, K.R., Jin, R., He, J.P., Kessler, R.C., Lee, S., Sampson, N.A., . . \& Zarkov, Z. (2011). Prevalence and correlates of bipolar spectrum disorder in the world mental health survey initiative. Archives of General Psychiatry, 68(3), 241-251.

Merikangas, K. R., He, J. P., Burstein, M., Swanson, S. A., Avenevoli, S., Cui, L., Benjet, C., Georgiades, K., \& Swendsen, J. (2010). Lifetime prevalence of mental disorders in U.S. adolescents: results from the National Comorbidity Survey Replication--Adolescent Supplement (NCS-A). Journal of the American Academy of Child and Adolescent Psychiatry, 49(10), 980989.

Merikangas, K. R., Nakamura, E. F., \& Kessler, R. C. (2009). Epidemiology of mental disorders in children and adolescents. Dialogues in Clinical Neuroscience, 11, 7-20.

Murray, L.K., Dorsey, S., Haroz, E., Lee, C., Alsiary, M.M., Haydary, A., Weiss, W.M. and Bolton, P. (2014). A Common Elements Treatment Approach for Adult Mental Health Problems in Low- and Middle-Income Countries. Cognitive and Behavioral Practice, 21(2), 111-123

Mynors-Wallis, L.M. (2005). Problem-solving treatment for anxiety and depression: A practical guide. Oxford University Press: Oxford.

Nezu, A.M. (1987). A problem solving formulation of depression: A literature review and proposal of a pluralistic model. Clinical Psychology Review, 7 121-144 
Perera, E.A.R., \& Kathriarchchi, S.T. (2011). Problem-solving counselling as a therapeutic tool on youth suicidal behavior in the suburban population in Sri Lanka. Indian Journal of Psychiatry $53(1)$.

Puig-Antich, J., Kaufman, J., Ryan, N.D., Williamson, D. E., Dahl, R.E., Lukens, E., Todak, G., Ambrosini, P., Rabinovich, H., \& Nelson, B. (1993). The psychosocial functioning and family environment of depressed adolescents. Journal of the American Academy of Child and Adolescent Psychiatry, 32, 244-253.

Reinecke, M.A., Ryan, N.E., \& DuBois, D.L. (1998). Meta-analysis of CBT for depression in adolescents: Dr. Reinecke et al. reply. Journal of the American Academy of Child and Adolescent Psychiatry, 37, 1006-1007.

Rohde, P., Lewinsohn, P. M., \& Seeley, J. R. (1991). Comorbidity of unipolar depression: II. Comorbidity with other mental disorders in adolescents and adults. Journal of Abnormal Psychology, 54, 653-660.

Sampson, M., Villarreal, Y., \& Rubin, A. (2016). A problem-solving therapy intervention for lowincome, pregnant women at risk for postpartum depression. Research on Social Work Practice, 26(3), 236-242.

Skeen, S., Laurenzi, C.A., Gordon, S.L., du Toit, S., Tomlinson, M., Dua, T., . . \& MelendezTorres, G.J. (2019). Adolescent Mental Health Program Components and Behavior Risk Reduction: A Meta-analysis. Pediatrics 144, 2, e20183488.

Smith, K. (2014). Mental health: A world of depression: A global view of the burden caused by depression. Nature, 515, 180-181.

Speckens, A.E.M. \& Hawton, K. (2005). Social Problem Solving in Adolescents with Suicidal Behaviour: A Systematic Review. Suicide and Life-Threatening Behaviour 35(4) 365-387

Spence, S.H., Sheffield, J.K., \& Donovan, C.L. (2003). Preventing adolescent depression: An evaluation of the problem solving for life program. Journal of Consulting and Clinical Psychology, 71(1), 3-13.

Sterne, J. A., Savović, J., Page, M. J., Elbers, R. G., Blencowe, N. S., Boutron, I., ... \& Emberson, J. R. (2019). RoB 2: a revised tool for assessing risk of bias in randomised trials. BMJ, 366(14898).

Stice, E., Rohde, P., Gau, J., \& Ochner, C. (2011). Relation of depression to perceived social support: Results from a randomized adolescent depression prevention trial. Behaviour Research and Therapy, 49(5), 361-366.

Weersing, V. R., \& Weisz, J. R. (2002). Community clinic treatment of depressed youth: Benchmarking usual care against CBT clinical trials. Journal of Consulting and Clinical Psychology, 70, 299-310. 
Weersing, V. R., Jeffreys, M., Do, M. T., Schwartz, K. T., \& Bolano, C. (2017). Evidence Base Update of Psychosocial Treatments for Child and Adolescent Depression. Journal of Clinical Child and Adolescent Psychology, 46(1), 11-43.

Weisz, J., Bearman, S.K., Santucci, L.C., \& Jensen-Doss, A. (2017). Initial test of a principleguided approach to transdiagnostic psychotherapy with children and adolescents. Journal of Clinical Child \& Adolescent Psychology, 46(1), 44-58.

Weisz, J., Gordis, E., Chu, B., McLeod, B., Updegraff, A., Southam-Gerow, M.A., .. \& Weiss, B. (2009). Cognitive-behavioral therapy versus usual clinical care for youth depression: An initial test of transportability to community clinics and clinicians. Journal of Consulting and Clinical Psychology, 77, 383-396.

Weisz, J.R., Kuppens, S., Eckshtain, D., Ugueto, A.M., Hawley, K.M., \& Jensen-Doss, A. (2013). Performance of evidence-based youth psychotherapies compared with usual clinical care: a multilevel meta-analysis. JAMA Psychiatry, 70(7), 750-761.

Weisz, J.R., Kuppens, S., Ng, M., Eckshtain, D., Vaughn-Coaxum, R.A., ... \& Fordwood, S. R. (2017). What five decades of research tells us about the effects of youth psychological therapy: A multilevel meta-analysis and implications for science and practice. American Psychologist, 72(2), 79-117.

Weisz, J.R., Kuppens, S., Ng, M., Vaughn-Coaxum, R.A., Ugueto, A.M., Eckshtain, D., \& Corteselli, K.A. (2019). Are psychotherapies for young people growing stronger? Tracking trends over time for youth anxiety, depression, attention-deficit/hyperactivity disorder, and conduct problems. Perspectives on Psychological Science, 14(2), 216-237.

Zisook, S., Lesser, I., Stewart, J. W., Wisniewski, S. R., Balasubramani, G. K., Fava, M., ... \& Trivedi, M. H. (2007). Effect of age at onset on the course of major depressive disorder. American Journal of Psychiatry, 164(10), 1539-1546. 